\title{
Interfacial Interactions During In Situ Polymer Imbibition in Nanopores
}

\author{
Chien-Hua Tu $\odot,{ }^{1}$ Jiajia Zhou $\odot,{ }^{2,3}$ Masao Doi $\odot,{ }^{3}$ Hans-Juergen Butt, ${ }^{1}$ and George Floudas $\odot^{1,4,5, *}$ \\ ${ }^{1}$ Max Planck Institute for Polymer Research, 55128 Mainz, Germany \\ ${ }^{2}$ School of Chemistry, Beihang University, Key Lab Bioinspired Smart Interfacial Science \& Technology of Ministry of Education, \\ Beijing 100191, China \\ ${ }^{3}$ Center of Soft Matter Physics and Its Applications, Beihang University, Beijing 100191, China \\ ${ }^{4}$ Department of Physics, University of Ioannina, 45110 Ioannina, Greece \\ ${ }^{5}$ University Research Center of Ioannina (URCI)_Institute of Materials Science and Computing, 45110 Ioannina, Greece
}

(Received 15 July 2020; accepted 21 August 2020; published 17 September 2020)

\begin{abstract}
Using in situ nanodielectric spectroscopy we demonstrate that the imbibition kinetics of cis-1,4polyisoprene in native alumina nanopores proceeds in two time regimes both with higher effective viscosity than bulk. This finding is discussed by a microscopic picture that considers the competition from an increasing number of chains entering the pores and a decreasing number of fluctuating chain ends. The latter is a direct manifestation of increasing adsorption sites during flow. At the same time, the longest normal mode is somewhat longer than in bulk. This could reflect an increasing density of topological constraints of chains entering the pores with the longer loops formed by other chains.
\end{abstract}

DOI: 10.1103/PhysRevLett.125.127802

Understanding that way that polymers penetrate in narrow pores is important for applications including separation of proteins with relevance in cell biology and the development of inkjet printing for commercial xerography. In general, placing macromolecules close to interfaces alter their conformational and dynamic properties $[1,2]$. Much of this behavior stems from polymer-substrate interactions [3-6]. Taming the strength of interfacial interactions especially under nonequilibrium conditions (e.g., during flow), will enable the design of polymer interfaces with controlled physical properties (viscosity, glass temperature as well as wettability and adhesion) of importance in applications (organic electronic devices, coatings, membranes).

A recent advancement in this field have been experiments by the so-called, nanofluidic method [7-10]. The method employs self-ordered nanoporous aluminum oxide (AAO) templates sputtered with a thin gold layer to transform the nanoporous membranes into capacitors where an ac field is applied along the pore axes (Fig. 1). When the openings of the pores are brought into contact with a polymer melt, capillary forces drive the polymer chains into the pores. The polymer increases the capacitance $C$ by way of the dielectric permittivity $\varepsilon$ of the penetrating polymer $(C \sim \varepsilon)$. The method offers

Published by the American Physical Society under the terms of the Creative Commons Attribution 4.0 International license. Further distribution of this work must maintain attribution to the author(s) and the published article's title, journal citation, and DOI. Open access publication funded by the Max Planck Society. simultaneous access to the kinetics of imbibition and to the molecular dynamics during flow at the segmental and possibly the chain length scales. Results have demonstrated the potential of the method to extract quantitative information on the viscosity experienced by the polymer during flow [7]. However, despite profound importance in applications, the pertinent polymer chain dynamics during flow have not been addressed either in theory or in experiment.

In this Letter we employ the nanofluidic method to follow the in situ polymer dynamics directly at the chain length scale by studying the evolution of normal modes in cis-1,4-polyisoprene (PI) during imbibition in AAO nanopores. The scientific question we address is if and how adsorption affects the imbibition kinetics of polymers. This question, despite being of importance in the design of

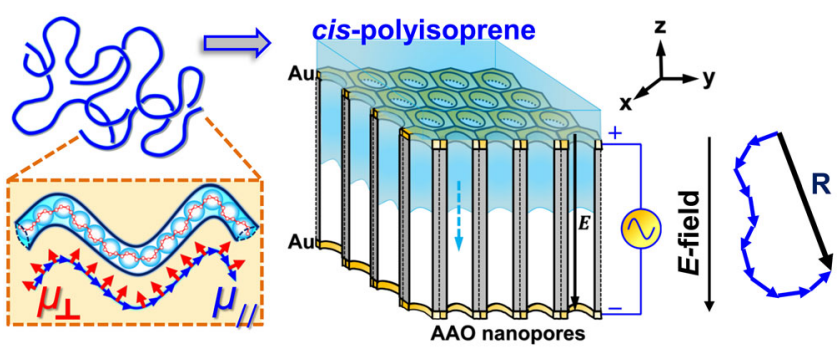

FIG. 1. Schematic of the AAO-sample geometry for the in situ imbibition of $c i s-1,4$-polyisoprene. In the upper part the dipole moment components along $\left(\mu_{\mid}\right)$and perpendicular $\left(\mu_{\perp}\right)$ to the chain contours are indicated. The direction of the $E$ field with respect to the end-to-end vector (thick arrow) is shown in the right. The electric filed is applied in the $z$ direction (flow direction), hence the $z$ component of the polarization is detected. 
membranes and devices, has not been addressed so far. The reason is that it requires precise measurements of polymer dynamics during flow. Results show that polymer imbibition proceeds in two time regimes with distinct changes in the dielectric strength of normal modes. We discuss this finding with the help of a microscopic picture that considers the competition from an increasing number of chains entering the pores and a decreasing number of fluctuating chains with time. The latter provide unambiguous evidence for increasing adsorption sites during flow.

The studied polyisoprene (PI) homopolymer had $M_{n}=$ 42410 and $M_{w}=44000 \mathrm{~g} \mathrm{~mol}^{-1}$ (PI-42k). AAO templates [11] with both ends open and thickness of $100 \mu \mathrm{m}$ were purchased from InRedox (Longmont, USA). Pore diameters and corresponding porosities were 100 $(24 \pm 3 \%), 40(12 \pm 2 \%)$, and $20 \mathrm{~nm}(11 \pm 2 \%)$, respectively. Two types of AAO surfaces were examined. One (the untreated) where templates were placed in an oven under vacuum at a temperature of $443 \mathrm{~K}$ for $8-10 \mathrm{~h}$ in order to remove the majority of $\mathrm{OH}$ groups from the $\mathrm{AAO}$ surface. Another one (the silanized) with a treated surface with trichloro $(1 \mathrm{H}, 1 \mathrm{H}, 2 \mathrm{H}, 2 \mathrm{H}$-perfluorooctyl)silane (for 5 min under vacuum followed by heating to $423 \mathrm{~K}$ in order to remove any excess silane). Dielectric spectroscopy (DS) measurements were carried out at different temperatures in the range from 183 to $393 \mathrm{~K}$, at atmospheric pressure, and for frequencies in the range from $10^{-2}-3 \times 10^{6} \mathrm{~Hz}$. For the in situ imbibition kinetic experiments, a gold layer (thickness $35 \mathrm{~nm}$ ) was sputtered on both sides of the AAO templates as top and bottom electrodes (Fig. 1). More details on material and methods can be found in the Supplemental Material [12].

Figure 2(a) provides fitting examples for bulk PI using a summation of two Havriliak and Negami (HN) functions $[13,14]$ following imbibition. The slower one corresponds to the longest normal mode whereas the faster to the summation of all higher (shorter) modes. We first note that the higher normal modes seem to approach the longest normal mode and second, the longest normal mode is longer than in the bulk. We will return to this point later.

The evolution of the dielectric loss curves at the length scale of the normal modes is depicted in Fig. 3(a) for AAO pores with diameter of $40 \mathrm{~nm}$. The radius of gyration of the polymer $R_{g}$ is smaller than the diameter of the pore $d$ $\left(2 R_{g} / d=0.35\right)$. The analysis of the dielectric loss curves provides the dielectric strength of the slower (longest normal mode) and faster (higher normal modes) modes [Fig. 3(b)]. It shows an initially increasing dielectric strength for both modes, scaling as $t^{1 / 2}$, up to $17000 \mathrm{~s}$. This increase is followed by a plateau at longer times (for the slower mode) or even a decrease (for the higher normal modes). Notably, the plateau is absent in the case of the silanized pores (Fig. S2, Supplemental Material [12]). In parallel, the imbibition length $L$ was measured ex situ by
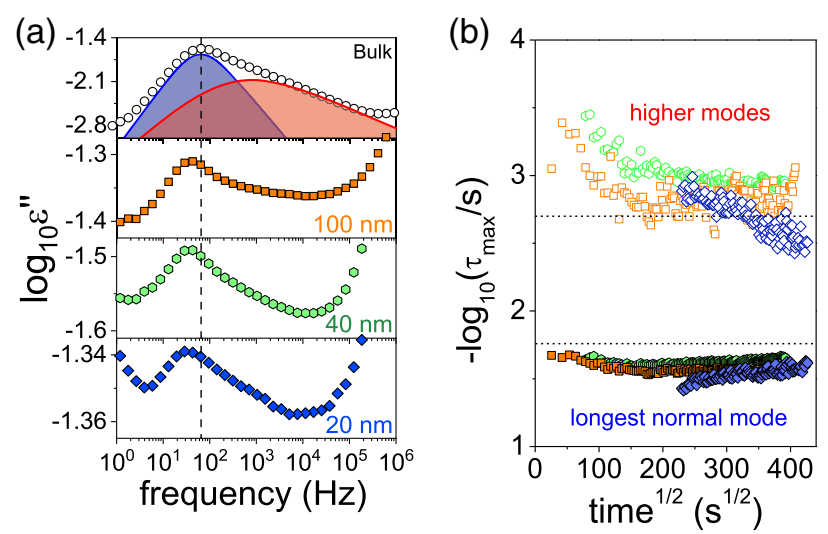

FIG. 2. (a) Dielectric loss curves of bulk and confined PI-42k within AAO pores with pore diameters of 100, 40, and $20 \mathrm{~nm}$ at $T=303 \mathrm{~K}$. The black dashed line indicates the peak position of the longest normal mode in bulk PI. Representative fit of the dielectric loss curve for the bulk PI-42k with a summation of two $\mathrm{HN}$ functions corresponding to the longest normal mode (blue shaded area) and the summation of shorter normal modes (red shaded area). (b) Evolution of the relaxation times for PI-42k during imbibition inside AAO nanopores as a function of the square-root of time. Same color codes as in (a): 100 (orange circles), 40 (green squares), and $20 \mathrm{~nm}$ (blue rhombi). The short dashed lines give the position of the longest normal mode and of the internal modes in the bulk polymer at the same imbibition temperature.

optical reflection microscopy and the result is shown in Fig. 3(c). Based on the Lucas-Washburn equation (LWE) developed for Newtonian liquids penetrating a cylindrical capillary of radius $R$, the imbibition length should scale as $[15,16]$ (a)

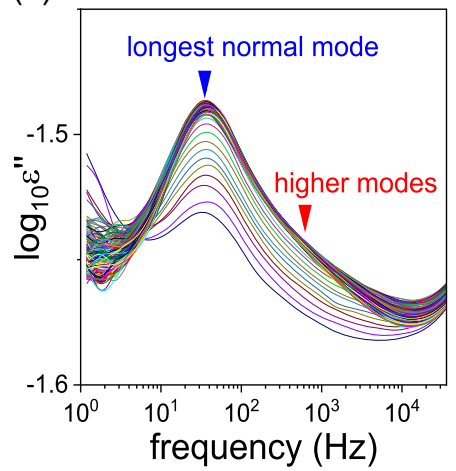

(b)

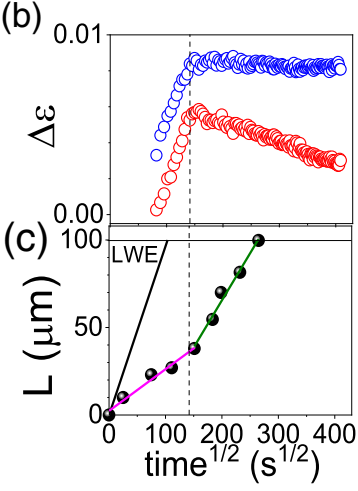

FIG. 3. (a) Evolution of the dielectric loss spectra of PI $42 \mathrm{k}$ during imbibition within AAO nanopores with pore diameter of $40 \mathrm{~nm}$ at $303 \mathrm{~K}$. (b) The extracted dielectric intensity as a function of the square root of imbibition time. (c) Imbibition lengths extracted via the optical reflection method at selected times. The dashed line is a guide for the eye. The extracted effective viscosity, $\eta_{\text {eff }}$, in the two regimes is $4660 \mathrm{~Pa}$ s (magenta) and $910 \mathrm{~Pa}$ s (green) for regimes I and II, respectively. The solid black line is the prediction of LWE. The calculation is based on the bulk viscosity $\eta_{0}($ bulk $)=285 \mathrm{Pas} ; \gamma_{L}=30.1 \mathrm{mN} / \mathrm{m}$; $\cos \theta=0.88 ; R=20 \mathrm{~nm}$. 


$$
L(t)=\left(\frac{\gamma R \cos \theta}{2 \eta}\right)^{1 / 2} \sqrt{t}
$$

Here, $\gamma$ is the surface tension, $\theta$ is the advancing contact angle, $R$ is the pore diameter, $\eta$ is the viscosity, and $t$ is the wetting time. Employing the bulk viscosity $\eta_{0}=285 \mathrm{Pas}$ (from rheology measurements), the measured surface tension $\gamma_{L}=30.1 \mathrm{mN} / \mathrm{m}$ and the measured advancing contact angle, $\theta=28^{\circ}$ we obtain the solid line in Fig. 3(c). The experimental results deviate from the LWE predictions. The $L$-versus- $\sqrt{t}$ plots [Fig. 3(c)] also show the two regimes. Up to $t=17000 \mathrm{~s}$ the polymer melt imbibes like a liquid with the effective viscosities of $4660 \mathrm{~Pa}$ s. For longer times the imbibition can be described by an effective viscosity of $910 \mathrm{~Pa}$ s. This suggests that the polymer penetrates the pores with a 16-fold and threefold higher viscosity than in the bulk. At the same time, the characteristic relaxation time corresponding to the longest normal mode [Fig. 2(b)] is slightly longer than in the bulk.

Higher effective viscosities than the bulk are not uncommon during polymer imbibition in nanopores [17-19]. Recently, Doi et al. [19] constructed a unified theory to describe the imbibition behavior of polymer melts in nanopores. Two different mechanisms were proposed: one is the dead-layer effect due to the adsorption of polymer segments. Strongly adsorbed polymer chains create a smaller effective pore radius $\left(R_{\text {effective }}<R_{\mathrm{AAO}}\right)$ giving rise to a higher effective viscosity $\left(\eta_{\text {effective }}>\eta_{\text {bulk }}\right)$. The other mechanism relies on the reptation of polymer chains under a pressure gradient. As pores reduce to a size comparable to the thickness of the dead layer (extreme confinement), polymer chain transport is achieved mainly by the reptation of free polymer chains in a network driven by the pressure gradient originating from the capillary force. This mechanism leads to the lower effective viscosity $\left(\eta_{\text {effective }}<\eta_{\text {bulk }}\right)$. The nonmonotonic behavior of the effective viscosity on pore size is the result of the competition between the two mechanisms. This effect found experimentally [17] was well captured by theory [19].

By contrasting the growing imbibition lengths [Fig. 3(c)] with the evolution of dielectric loss [Fig. 3(b)] we come to, at first site, a surprising finding: Although in regime I the polymer advances within the pores following the LWE (albeit with a higher effective viscosity), in regime II, the polymer continues to advance under a constant (or even slightly decreasing) dielectric strength. To better understand this result we need to comment on the microscopic factors that influence the dielectric strength for the segmental and normal mode processes. The corresponding $\Delta \varepsilon$ 's for the segmental and normal mode processes are [20-27]

$$
\Delta \varepsilon_{\text {segmental }}=\frac{1}{3 \varepsilon_{0}} F g \frac{\mu_{s}^{2}}{k_{B} T} \frac{N_{A}}{V},
$$

$$
\Delta \varepsilon_{\text {longest n.m. }}=\frac{4 \pi N_{A} \mu_{N M}^{2}}{3 k_{B} T} \frac{\left\langle r^{2}\right\rangle}{M_{P I}} .
$$

In Eq. (2), for the segmental relaxation $F$ is the local field, $g$ the Kirkwood-Fröhlich correlation factor, $\mu_{s}$ the dipole moment per molecule, and $N_{A}$ Avogadro's number. Evidently, the dielectric strength for the segmental mode being proportional to the number density of dipoles, is an increasing function of imbibition time up to the complete pore filling. This has been confirmed by following the evolution of the segmental process in PI (at a lower temperature) and in another polymer [poly (n-butyl methacrylate) [7] ]. Hence, the evolution of the segmental process follows approximately the predictions from Eq. (2).

In contrast, the dielectric strength of the chain modes relates to the relaxation of the end-to-end vector, $\left\langle r^{2}\right\rangle$, the dipole moment per contour length, $\mu_{\mathrm{NM}}$, and the chain molecular weight, $M_{\mathrm{PI}}$. The former is prone to chain configurations next to the pore wall (Fig. 4). For example, adsorbed polymer segments with a fixed train configuration next to the walls do not fluctuate in time and hence do not contribute to the dielectric strength of the chain relaxation. Likewise, segments in looped configurations do not contribute to the dielectric strength as both ends are fixed at the pore walls. Only segments located in the tails can fluctuate in time and hence contribute to the dielectric strength of the chain relaxation. The data shown in Fig. 3 provide unambiguous evidence than only a portion of chains (i.e., subchains) fluctuate in time during the imbibition process. This should be contrasted with the silanized pores where the dielectric strength is increasing up to complete pore filling. Surface treatment reduces adsorption sites effectively acting as lubricant for the chains (see Fig. S2, Supplemental Material [12]). However, it is puzzling that the obtained relaxation time of the chain relaxation (Fig. 2) is not faster-as it would be expected for shorter chain configurations associated with the free ends [27].

Below we provide a plausible microscopic scenario (with the help of Fig. 4) that attempts to reconcile all experimental findings. The figure depicts only a few chains and shows the imbibition process at the beginning (corresponding to regime I) and at later stages (regime II) of the imbibition process. At the beginning, only a small portion of chains are in contact with the pore walls forming trains, loops and some tails (e.g., chain in red). Despite the small fraction of adsorbed segments, the effective pore diameter is reduced and the overall viscosity is increased reflecting the effect of the dead layer. However, the majority of chains are not in contact with the pore walls and hence there exist fluctuations of the end-to-end vector (e.g., chains in green, blue, and yellow). As time progresses within regime I $\left(t^{1 / 2}<150 \mathrm{~s}^{1 / 2}\right)$ more polymer chains enter the pores and the dielectric strength increases [Eq. (3)]. At longer times, within regime II, more segments are adsorbed at the pore walls creating more train and longer loop configurations 


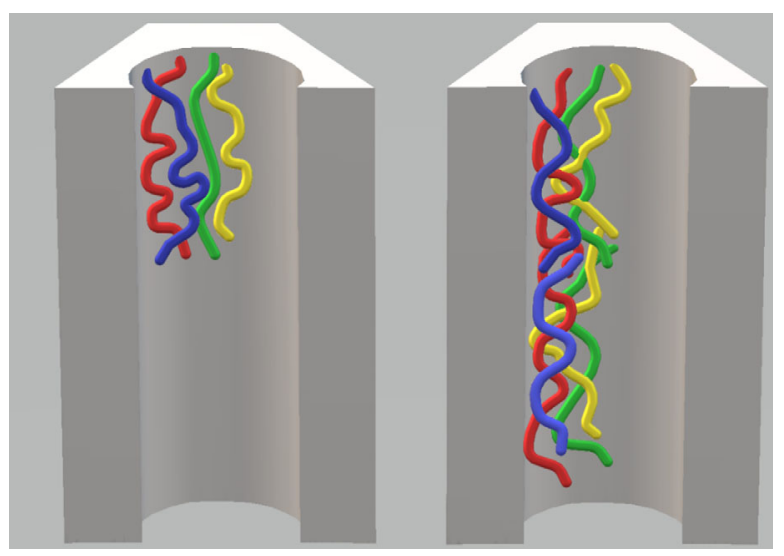

FIG. 4. Schematic of PI imbibition in native nanopores. Four colored chains are shown at initial (left) and additional chains at later stages (right). Initially only a small number of segments corresponding to the red chain are adsorbed to the walls forming some trains, loops and tails. At latter stages, additional chains have segments in the vicinity of the pore walls and inner chains (e.g., yellow chain) are more entangled with the loops of the adsorbed chains (red or blue chains).

that have as an effect the reduction in the dielectric strength for the normal modes.

The kinetics of irreversible adsorption were first studied theoretically for functionalized polymers in solution [3] and subsequently generalized for thin polymer films (where confinement is one dimensional) by evaluating the thickness of the adsorbed layer [4-6]. It was found that adsorption follows two time regimes; at short annealing times, the thickness $\left(h_{\text {ads }}\right)$ increases linearly with time due to the available substrate surface scaling as ( $h_{\text {ads }} \sim N^{1 / 2} \sim R_{g}, N$ is the degree of polymerization). At longer times, the available surface sites are reduced and chains adsorb by loops with a length that increases in time. The thickness of the adsorbed layer in this case increases slower with time with a logarithmic dependence. As the subchains forming the loops cannot fluctuate, the loops inevitably become dielectrically inactive. As a result, the dielectric strength of the normal modes will decrease with time [4]. These effects, obtained under quasiequilibrium conditions, will also affect the dielectric strength of the normal modes in the present case, e.g., during polymer flow. Interestingly, the fast-growing initial dependence and the final slower logarithmic dependence is reminiscent to the square-root time dependence of the imbibition length [Eq. (1)] but this point needs to be explored further.

Despite the similarities with 1D confinement there exist, however, a fundamental difference. In the present case, the near constancy of the dielectric strength is a composite effect owing to an increasing number of chains within the pores and a decreasing number of fluctuating chains [Fig. 3(b)]. Interestingly, the crossover time from Fig. 3(b) corresponds to a timescale much longer than the terminal relaxation time of the bulk polymer (see Fig. S7,
Supplemental Material [12]). This provides additional support to the fact that other-longer-timescales are involved in the adsorption process. Adsorption, apart from chain diffusion towards the interphase, involves continuous conformational pathways of the chains towards minimizing their free energy.

The existence of a somewhat slower longest normal mode (Fig. 2) within regime II in the native AAO nanopores is more difficult to explain. It could associate with an increasing density of topological constraints (entanglements). New chains entering the pores (e.g., yellow chains in Fig. 4) are topologically constrained by the increased numbers of longer loops. This can explain the longer characteristic time for the normal modes during polymer flow (Fig. 2). Notably, this effect is absent in the case of silanized AAO (see Fig. S3, Supplemental Material [12]). In the absence of adsorption sites long loops are not formed, the chains do not experience extra topological constraints and imbibe with the same timescale as in bulk.

These results will depend on the degree of confinement (e.g., $2 R_{g} / d$ ). For the same PI-42k entering $100 \mathrm{~nm}$ pores (see Fig. S3, Supplemental Material [12]) where $2 R_{g} / d \sim 0.14$, the imbibition process has also two regimes with enhanced viscosity than in bulk, however, stage II has now the higher viscosity. In addition, part of the findings could be attributed to changes in glass temperature, $T_{g}$, under confinement [28-33]. Having this in mind we studied the segmental dynamics following the in situ imbibition (by cooling and subsequent heating runs (see Fig. S4, Supplemental Material [12]). In agreement with earlier studies the segmental dynamics become bimodal below some "critical" temperature $\left(T_{c} \sim 226 \mathrm{~K}\right)$ [31-33]. The high $T_{g}$ is near (slightly above) the bulk $T_{g}$ whereas the lower $T_{g}$ depends on the degree of confinement. The shift in $T_{g}$ from the bulk value in the latter case can be parametrized as $\Delta T_{g} \sim-54\left(2 R_{g} / d\right)$ (see Fig. S5, Supplemental Material [12]) for a range of PI molecular weights and degrees of confinement. Changes in $T_{g}$ under confinement have been discussed in terms of increasing interfacial energy for a number of different polymers $[28,29]$.

Lastly, we compare the current findings with the results from a rheodielectric experiment on the same polymer [34] where the dielectric response was followed during shear. It was shown that the latter is much less sensitive to shear as compared to the strong shear-thinning behavior in the viscoelastic response. Differences in the viscoelastic and dielectric responses were discussed in terms of isochronal orientational cross-correlation of segments under fast shear. We note two differences from the present case. First, viscoelasticity is less important for flow within channels. From the data depicted in Fig. 3(c), we can extract effective shear rates as $\dot{\gamma}=v / R(\sim 0.5 \mathrm{rad} / \mathrm{s}), v$ is the velocity, $R$ is the pore radius. At such low shear rates, the viscosity that is experienced by the polymer during flow is the zero-shear 
viscosity (see Fig. S7, Supplemental Material [12]). Second, the measured dielectric strength $(\Delta \varepsilon)$ reflects the chain dimension $\left\langle R_{z}^{2}\right\rangle$ in the flow direction (Fig. 1) as opposed to the shear gradient direction probed in rheodielectrics.

In conclusion, the imbibition kinetics of entangled cis-1,4-PI was studied, for the first time during flow, by in situ nanodielectric spectroscopy, under conditions of confinement $\left(0.14<2 R_{g} / d<0.35\right)$ and as a function of pore surface treatment. For native AAO pores, imbibition follows two time regimes both with increased effective viscosity relative to bulk. Within the later stages the dielectric strength of the longest normal mode remains constant despite the fact that more chains are entering the pores. We have discussed this finding by a microscopic picture that considers the competition from an increasing number of chains entering the pores (that increases the dielectric strength) and a decreasing number of fluctuating chains (that decreases the dielectric strength). At the same time, the longest normal mode is somewhat longer than in bulk within native pores but not in silanized pores. This could reflect an increased density of topological constraints with time in the former case.

In situ dielectric spectroscopy provides us with access to length scales and timescales (from the local segmental to internal chain modes, and to the longest normal mode) under nonequilibrium conditions (during flow) never considered before. The method and current results provided unambiguous evidence for growing interfacial interactions. Taming the strength of interfacial interactions under such nonequilibrium conditions could help towards designing polymer interfaces with controlled physical properties.

This research was supported by the Hellenic Foundation for Research and Innovation (H. F. R. I.) under the "First Call for H. F. R. I. Research Projects to support Faculty members and Researchers and the procurement of high-cost research equipment grant" (Project No. 183).

* Corresponding author. gfloudas@uoi.gr

[1] S. Granick, Motions and relaxations of confined liquids, Science 253, 1374 (1991).

[2] Advances in Dielectrics, in, Dynamics in Geometrical Confinement, edited by F. Kremer (Springer, Berlin, 2014).

[3] C. Ligoure and L. Leibler, Thermodynamics and kinetics of grafting end-functionalized polymers to an interface, J. Phys. 51, 1313 (1990).

[4] S. Napolitano and M. Wübbenhorst, The lifetime of the deviations from bulk behaviour in polymers confined at the nanoscale, Nat. Commun. 2, 260 (2011).

[5] C. Housmans, M. Sferrazza, and S. Napolitano, Kinetics of irreversible chain adsorption, Macromolecules 47, 3390 (2014).

[6] D. N. Simavilla, A. Panagopoulou, and S. Napolitano, Characterization of adsorbed polymer layers: preparation, determination of the adsorbed amount and investigation of the kinetics of irreversible adsorption, Macromol. Chem. Phys. 219, 1700303 (2018).

[7] C.-H. Tu, M. Steinhart, H.-J. Butt, and G. Floudas, In situ monitoring of the imbibition of poly(n-butyl methacrylates) in nanoporous alumina by dielectric spectroscopy, Macromolecules 52, 8167 (2019).

[8] A. Serghei, D. Chen, D. H. Lee, and T. P. Russell, Segmental dynamics of polymers during capillary flow into nanopores, Soft Matter 6, 1111 (2010).

[9] A. Serghei, J. L. Lutkenhaus, D. F. Miranda, K. McEnnis, F. Kremer, and T. P. Russell, Density fluctuations and phase transitions of ferroelectric polymer nanowires, Small 6 , 1822 (2010).

[10] A. Serghei, W. Zhao, X. Wei, D. Chen, and T. P. Russell, Nanofluidics with phase separated block-copolymers: Glassy dynamics during capillary flow, Eur. Phys. J. Spec. Top. 189, 95 (2010).

[11] H. Masuda and K. Fukuda, Ordered metal nanohole arrays made by a two-step replication of honeycomb structures of anodic alumina, Science 268, 1466 (1995).

[12] See Supplemental Material at http://link.aps.org/ supplemental/10.1103/PhysRevLett.125.127802 for further details on the technique, sample preparation, and data evaluation; additional in situ data as a function of surface treatment, results on the segmental dynamics following imbibition, and the $T_{g}$ dependence on confinement.

[13] F. Kremer and A. Schönhals, Broadband Dielectric Spectroscopy (Springer, Berlin, 2002).

[14] G. Floudas, M. Paluch, A. Grzybowski, and K. L. Ngai, , Molecular Dynamics of Glass-Forming Systems: Effects of Pressure (Springer, Berlin, 2011).

[15] R. Lucas, Ueber das zeitgesetz des kapillaren aufstiegs von flüssigkeiten, Colloid Polym. Sci. 23, 15 (1918).

[16] E. W. Washburn, The dynamics of capillary flow, Phys. Rev. 17, 273 (1921).

[17] Y. Yao, S. Alexandris, F. Henrich, G. Auernhammer, M. Steinhart, H.-J. Butt, and G. Floudas, Complex dynamics of capillary imbibition of poly (ethylene oxide) melts in nanoporous alumina, J. Chem. Phys. 146, 203320 (2017).

[18] Y. Yao, Y. Suzuki, J. Seiwert, M. Steinhart, H. Frey, H.-J. Butt, and G. Floudas, Capillary Imbibition, crystallization, and local dynamics of hyperbranched poly (ethylene oxide) confined to nanoporous alumina, Macromolecules 50, 8755 (2017).

[19] Y. Yao, H.-J. Butt, G. Floudas, J. Zhou, and M. Doi, Theory on capillary filling of polymer melts in nanopores, Macromol. Rapid Commun. 39, 1800087 (2018).

[20] K. Adachi and T. Kotaka, Dielectric normal mode relaxation, Prog. Polym. Sci. 18, 585 (1993).

[21] K. Adachi and T. Kotaka, Dielectric normal mode process in undiluted cis-polyisoprene, Macromolecules 18, 466 (1985).

[22] D. Boese, F. Kremer, and L. J. Fetters, Molecular dynamics in linear and multiarmed star polymers of cis-polyisoprene as studied by dielectric spectroscopy, Macromolecules 23, 1826 (1990).

[23] D. Boese and F. Kremer, Molecular dynamics in bulk cispolyisoprene as studied by dielectric spectroscopy, Macromolecules 23, 829 (1990). 
[24] A. Schönhals, Relation between main and normal mode relaxations for polyisoprene studied by dielectric spectroscopy, Macromolecules 26, 1309 (1993).

[25] G. Floudas, C. Gravalides, T. Reisinger, and G. Wegner, Effect of pressure on the segmental and chain dynamics of polyisoprene. Molecular weight dependence, J. Chem. Phys. 111, 9847 (1999).

[26] G. Floudas, S. Paraskeva, N. Hadjichristidis, G. Fytas, B. Chu, and A. N. Semenov, Dynamics of polyisoprene in star block copolymers confined in microstructures: A dielectric spectroscopy study, J. Chem. Phys. 107, 5502 (1997).

[27] L. Petychakis, G. Floudas, and G. Fleisher, Chain dynamics of polyisoprene confined in porous media. A dielectric spectroscopy study, Europhys. Lett. 40, 685 (1997).

[28] S. Alexandris, P. Papadopoulos, G. Sakellariou, M. Steinhart, H.-J. Butt, and G. Floudas, Interfacial energy and glass temperature of polymers confined to nanoporous alumina, Macromolecules 49, 7400 (2016).

[29] A. Talik, M. Tarnacka, I. Grudzka-Flak, P. Maksym, M. Geppert-Rybczynska, K. Wolnica, E. Kaminska, K. Kaminski, and M. Paluch, The role of interfacial energy and specific interactions on the behavior of poly (propylene glycol) derivatives under 2D confinement, Macromolecules 51, 4840 (2018).

[30] S. Alexandris, G. Sakellariou, M. Steinhart, and G. Floudas, Dynamics of unentangled cis-1,4-Polyisoprene confined to nanoporous alumina, Macromolecules 47, 3895 (2014).

[31] C. Politidis, S. Alexandris, G. Sakellariou, M. Steinhart, and G. Floudas, Dynamics of entangled cis-1,4-polyisoprene confined to nanoporous alumina, Macromolecules 52, 4185 (2019).

[32] M. Tarnacka, K. Kaminski, E. U. Mapesa, E. Kaminska, and M. Paluch, Studies on the temperature and time induced variation in the segmental and chain dynamics in poly (propylene glycol) confined at the nanoscale, Macromolecules 49, 6678 (2016).

[33] E. C. Glor, G. V. Angrand, and Z. Fakhraai, Exploring the broadening and the existence of two glass transitions due to competing interfacial effects in thin, supported polymer films, J. Chem. Phys. 146, 203330 (2017).

[34] H. Watanabe, S. Ishida, and Y. Matsumiya, Rheodielectric behavior of entangled cis-polyisoprene under fast shear, Macromolecules 35, 8802 (2002). 